\title{
The cell wall-associated kinases, WAKs, as pectin receptors
}

\section{Bruce D. Kohorn* and Susan L. Kohorn}

Biology Department, Bowdoin College, Brunswick, ME, USA

\section{Edited by:}

Seth DeBolt, University of Kentucky, USA

\section{Reviewed by:}

Alison Roberts, University of Rhode Island, USA

Darby M. Harris, Cornell University, USA

\section{${ }^{*}$ Correspondence:}

Bruce D. Kohorn, Biology

Department, Bowdoin College, 6500

College Station, Brunswick, ME, USA.

e-mail: bkohorn@bowdoin.edu
The wall-associated kinases, WAKs, are encoded by five highly similar genes clustered in a 30-kb locus in Arabidopsis. These receptor-like proteins contain a cytoplasmic serine threonine kinase, a transmembrane domain, and a less conserved region that is bound to the cell wall and contains a series of epidermal growth factor repeats. Evidence is emerging that WAKs serve as pectin receptors, for both short oligogalacturonic acid fragments generated during pathogen exposure or wounding, and for longer pectins resident in native cell walls. This ability to bind and respond to several types of pectins correlates with a demonstrated role for WAKs in both the pathogen response and cell expansion during plant development.

Keywords: WAKs, pectin, cell wall, receptor kinase, pathogen response, cell expansion

\section{INTRODUCTION}

The wall-associated kinases, or WAKs, are receptor-like kinases that are linked to the pectin fraction of the cell wall, and have a cytoplasmic protein kinase domain. WAKs are required for cell expansion, are involved in the pathogen response, and their expression is activated by numerous environmental stimuli. Recent work supports the idea that WAKs are receptors for both pectin in the cell wall, and for pectin fragments, oligogalacturonic acids (OGs), generated during some pathogen attacks. The history of WAK isolation, and their characterization that led to the idea that WAKs are OG and pectin receptors, follows.

\section{IDENTIFICATION OF WAKs}

The WAKs were identified though the chance isolation of their cDNAs that subsequently led to the recognition of their significance to the cell wall (Kohorn et al., 1992; He et al., 1996; Kohorn, 2000, 2001). The WAKs are defined as cell wall-associated receptors by several key observations. WAKs could only be extracted from cells by boiling in detergent and reductant and appeared cross-linked to insoluble material. In addition, electron micrographs with specific WAK-kinase antiserum showed WAK protein in the cell wall and on the plasma membrane. Yet protease treatment of protoplasts and Western blots revealed that the kinase domain was cytoplasmic, and the receptor crossed the membrane such that the epidermal growth factor (EGF) domain is placed in the extracellular space (He et al., 1996). The five WAK genes are tightly clustered in tandem in a 30-kb locus on chromosome 1 (He et al., 1999), their encoded kinase domains are $85 \%$ identical, and the extracellular regions shows up to $65 \%$ identity (He etal., 1999). However, all WAKs contain the same conserved spacing of cysteine residues in the extracellular domain, the hallmark of the EGF repeat of metazoans (Sampoli Benitez and Komives, 2000).

\section{WAK-LIKE GENES}

There are at least 21 other genes for receptor-like kinases that contain EGF repeats in Arabidopsis, and these have been coined
WAK-like or WAKL genes (Verica and He, 2002; Verica et al., 2003). In crop plants, the WAKLs have greatly expanded in number and are often present in several large clusters. The expansion and size of this family indicates their importance, and some have suggested they play a role in pathogen resistance (Verica and He, 2002; Zhang et al., 2005). Stronger evidence for this idea is provided by the observation that dominant mutant alleles of WAKLs in Arabidopsis provide resistance to Fusarium, and a rice WAKL allele is less sensitive to rice blast disease (Diener and Ausubel, 2005; Li et al., 2009). Despite their importance and interesting biology, there is insufficient evidence to conclude that the WAKL proteins are wall-associated. The WAKLs instead may describe a class of plant receptor that is defined by the presence of EGF repeats, and whose additional domains and perhaps function has diverged from the WAKs. One might expect, therefore, their functions to be accordingly diverse.

\section{WAKs ARE REQUIRED FOR CELL EXPANSION}

Several lines of evidence point to a role of WAKs in cell expansion. Antisense WAK RNA to the conserved WAK-kinase domain can be induced using the Dex system, and this leads to a $50 \%$ reduction in WAK protein levels and a smaller cell size, rather then fewer cells. Thus, WAKs are required for cell elongation (Anderson etal., 2001; Lally et al., 2001; Wagner and Kohorn, 2001). A WAK2 null allele, wak2-1, causes a loss of cell expansion in roots, but only under limiting sugar and salt conditions (Kohorn et al., 2006b). Individual loss of function alleles in any of the four other WAKs do not result in an obvious phenotype, and unfortunately genetic analysis of the Arabidopsis WAKs has been greatly hampered by the fact that all five genes lie in a $30-k b$ cluster, making it difficult to create double or triple mutants of the isoforms (He et al., 1999).

A survey of enzyme activity showed that in roots of the wak2-1 null mutants there was a reduction in vacuolar invertase activity. Since invertase RNA levels are also affected, it suggests that WAK2 can regulate invertase at the transcriptional level. These results support a model where WAKs regulate cell 
expansion through a control of sugar concentration and thus perhaps turgor control. Consistent with this model is the observation that the wak2-1 phenotype could also be rescued by the expression of sucrose phosphate synthase which alters sugar sinks (Kohorn et al., 2006b). What remained was to establish how WAKs might be sensing the cell wall to effect the turgor regulated cell expansion.

\section{WAKs BIND TO PECTIN}

Wall-associated kinases are plasma membrane receptors, appear to be cross-linked to cell wall material, and can be seen by electron microscopy to lie in the cell wall. Pectinase, but not cellulase or other cell wall degrading enzymes release WAKs from the cell wall material. This led to the initial suggestion that WAKs are bound to pectins (Anderson et al., 2001; Wagner and Kohorn, 2001). Indeed, extracted WAK protein is still bound to a pectin epitope on denaturing gels, suggesting a covalent binding to a pectic fragment (Anderson et al., 2001; Wagner and Kohorn, 2001). The purified extracellular domains of WAK1 or 2 bind to pectin in vitro, and have a higher affinity for de-esterified pectin than to esterified molecules that did not have as negative a charge. Moreover, short pectin fragments of degree of polymerization (dp) 9-15 effectively competed with longer pectins for WAK binding (Decreux and Messiaen, 2005; Kohorn et al., 2006b, 2009). Mutation of the positively charged residues in WAK1 to neutral amino acids lead to a loss of binding to de-esterified pectin suggesting that the interaction was in part charge based (Decreux et al., 2006). These in vitro results provided confirmation of a WAK-pectin association, but also a puzzle as to why the WAK isolated from plants after pectinase treatment, had a covalent attachment to pectin. Moreover, it raised the question as to what type of pectin WAK was binding to and was perhaps responsive to in plants. In vitro, both WAK1 and 2 bind to a variety of pectins including polymers of homogalacturonan (HA), OGs, and to rhamnogalacturonans (RG) I and II (Kohorn et al., 2009). The common feature of these molecules is presence of a galacturonic acid backbone, and this is therefore the predicted target of WAKs. However, the backbone of RGII is not HA, suggesting that the binding requirements are not to a simple polymer of HA, but perhaps the presence galacturonic acid.

An analysis of WAK-GFP expression noted that WAK is crosslinked into a detergent-insoluble complex within the Golgi which contains pectin, and this is independent of fucose modification or cellulose synthesis. Thus, the assembly and crosslinking of WAKs may begin at an early stage within a cytoplasmic compartment rather than in the cell wall itself, and is coordinated with synthesis of surface cellulose (Kohorn et al., 2006a).

The biological activity of pectin fragments, or OGs, has been recorded for many years, with suggestions of a role in both defense and stress responses, and in developmental processes (Yamazaki et al., 1983; Willats et al., 2001; Mohnen, 2008; Harholt et al., 2010). Pathogen invasion, physical wounding, and herbivory have the ability to generate OGs in the cell wall and it has been suggested by many that there was a specific receptor for these OGs. WAKs may indeed be this receptor, but how it distinguishes OGs from nascent pectins is not clear. The in vitro binding data raise several other interesting questions. Pectin can be de-esterified once secreted into the cell wall by a family of methyl esterases, revealing a negative charge that permits a calcium-induced crosslinking with other pectins, and perhaps other carbohydrates and proteins in the wall (Bosch and Hepler, 2005; Krichevsky et al., 2007). Numerous reports have also documented an unequal distribution of esterified and de-esterified pectins in a variety of cell types, and correlations between the degree of esterification and cell enlargement have been observed (Willats et al., 2001; Mohnen, 2008; Caffall and Mohnen, 2009; Wolf et al., 2009; Harholt et al., 2010). How the differential binding of WAKs to esterified and de-esterified pectin relates to the unequal distribution has yet to be explored. WAKs in most cells appear to be uniformly distributed (He et al., 1996) as viewd by low resolution microscopy, but further imaging is needed. In the emerging pollen tube WAK epitopes are only detected near the tip. WAKs are present in the growing pollen tube, and its relationship to pectin deposition and directional growth also needs more exploration.

\section{WAKs ARE RESPONSIVE TO PECTINS}

Several recent lines of evidence indicate that the binding of pectin to WAKs activates several signaling pathways. The first is that pectin treatment of protoplasts causes the induction and repression of hundreds of genes involved in cell wall biogenesis and stress responses, and this response is blocked in cells lacking WAK2 (Kohorn etal., 2006b, 2009). An invertase promoterRFP reporter in protoplasts was used to show that pectin can activate invertase expression, but not in a wak2-1 null. While there may be additional proteins in the membrane that mediate the pectin response, the data are consistent with WAK1 and 2 serving as pectin receptors. This suggestion received more support from experiments in which the WAK1 extracellular domain was fused to a different cytoplasmic kinase, EFR, creating a novel chimeric receptor. The EFR kinase domain is known to activate ROS accumulation, a rise in ethylene levels, and a change in gene expression and these served as a recordable down stream pathway for the WAK extracellular domain (Brutus et al., 2010). Indeed, OGs activated a pathway downstream of the chimeric WAK-EFR kinase, when the chimera was transiently expressed in tobacco leaves. While these experiments entail a chimeric and hence novel receptor in a heterologous tissue, and the downstream readouts are also in part activated by both the native EFR kinase and the WAK kinase, they do support the idea that WAKs are an OG receptor. Detailed structural analysis and kinase activation studies are needed to understand how OG, or longer pectins can bind to WAKs to actually activate the protein kinase domain. These reports do not address the question of how WAKs might distinguish longer pectin polymers present in the cell wall from newly generated and bioactive OGs. Indeed, the ability to bind different types of pectins mirrors the bi-functional role of WAKs in pathogenesis and normal development, and is discussed further below.

\section{PROTEIN LIGANDS?}

In addition to OGs and pectins, WAK may also bind to glycinerich proteins (GRPs) of the cell wall. GRP3 interacts in a yeast two-hybrid assay with an extracellular domain of WAK1 that does not contain the EGF repeats (Park et al., 2001). The WAK-GRP3 
interaction is specific to WAK1 but not WAK2 or 4 in the yeast two-hybrid assay (Anderson et al., 2001). GRPs are a large family of secreted proteins in Arabidopsis and it is possible that different family members interact with different WAKs (Anderson et al., 2001; Mousavi and Hotta, 2005; Mangeon et al., 2010). It is believed that GRPs are structural cell wall proteins (Ringli et al., 2001), and since they might bind WAKs they may also have a signaling capacity. Both GRP3 and WAK1 can be isolated in the same $450 \mathrm{kDa}$ complex in Arabidopsis extracts, yet this result is surprising given that WAK1 is normally not detected as a water soluble protein, and further analysis is needed to confirm this result (Park et al., 2001). Blue native gel analysis of protein complexes fails to identify this WAK-GRP complex, and indeed the relationship between GRP, pectin, and WAKs warrants more exploration (Anderson et al., 2001).

\section{WAKs AND THE STRESS RESPONSE}

Wall-associated kinases are clearly required for cell expansion but they are also involved in the response to pathogen and stress. WAK expression is induced by wounding, pathogen infection, and by many stresses such as ozone and heavy metals (He et al., 1998; Anderson et al., 2001; Kohorn, 2001; Wagner and Kohorn, 2001; Sivaguru et al., 2003). This induced expression, however, is late in the signaling response and does not necessarily indicate a role for the WAK receptor itself. Dominant alleles of WAK1 that lack the extracellular domain did cause a resistance to high levels of salicylate, and antisense WAK1 plants had increased sensitivity to salicylate (He et al., 1998). While it was not clear how WAKs were involved, the work did establish a connection with pathogenesis, and reinforced the need to explore the role that pathogen-induced OGs might have in regulating WAKs. More recently a direct role for WAKs in the pathogen response was established. A dominant allele of WAK2, WAK2cTAP, was observed to cause ectopic lesions, ROS accumulation, curling of leaves, and stunted growth, all hallmarks of a pathogen effect, yet in the absence of pathogen (Kohorn et al., 2009,2011). WAK2cTAP alleles mutant in either the pectin binding domain, the EGF region, or in the catalytic kinase site do not induce these phenotypes indicating that active receptor function is required. Moreover, the WAK2cTAP receptor is still responsive to pectin stimulation, and appears to be a hyper-activated allele (Kohorn et al., 2011).

\section{MPKs}

MPK3 activity is elevated in cells treated with long polymers of pectin (Kohorn etal., 2009), and OGs have been shown to activate MPK3 (Moscatiello et al., 2006; Andreasson and Ellis, 2010). The idea that WAKs serve as pectin receptors is supported by the observation that plants homozygous for the null allele wak2-1 showed a reduction in the activation of MPK3 relative to wild-type (Kohorn et al., 2009). Moreover, plants expressing the WAK2cTAP dominant allele have a constitutively active MPK3 and 6. A WAK2cTAP allele in a mapk3-/- background had more severe growth defects than WAK2cTAP alone, supporting the concept that MPK3 is required for downstream WAK2 signaling (Kohorn et al., 2009). In contrast, a mpk6 null allele is able to suppress the effects of this dominant WAK2 mutation, thus distinguishing MPK3 and MPK6 whose activity previously was thought to be redundant (Andreasson and Ellis, 2010; Kohorn et al., 2011). These results suggest that WAKs can activate different downstream pathways depending upon the state of the wall, and a model is presented below. However, an analysis of other MPKs is still needed to fully understand WAK-MPK signaling.

\section{WAKs DISTINGUISH THE STATE OF PECTIN: A MODEL}

Wall-associated kinases are bound to pectin in native cell walls and their activity is required for normal cell expansion, yet OGs also bind to WAKs and mediate a response to pathogens and wounding (Kohorn et al., 2006b, 2009, 2011; Denoux et al., 2008; Brutus et al., 2010). Taken together, the results are consistent with a model presented in Figure 1. Here the type and concentration of pectin present in the wall could lead to a WAK-dependent activation of different signaling pathways. Unchallenged but expanding walls would preferentially activate via WAKs a cell expansion path that includes MPK3 (that other receptors are signaling via MPK3 and 6 is indicated by additional arrows). When OGs are generated by a wall disturbance, the WAKs may alter their signaling path to help effect the stress response by now also activating MPK6 and a new downstream response (Figure 1, right side). That the in vitro binding assays reveal a higher binding affinity of OGs than longer polymers for WAK (Decreux and Messiaen, 2005; Decreux et al., 2006) suggests a mechanism by which WAKs can switch from binding the native cell wall pectin to OGs, thus distingushing types of pectin. Differential activation by various pectins might be achieved by the specific pectin affinity of an individual receptor, or perhaps combinations of WAKs with as yet unidentified partners.

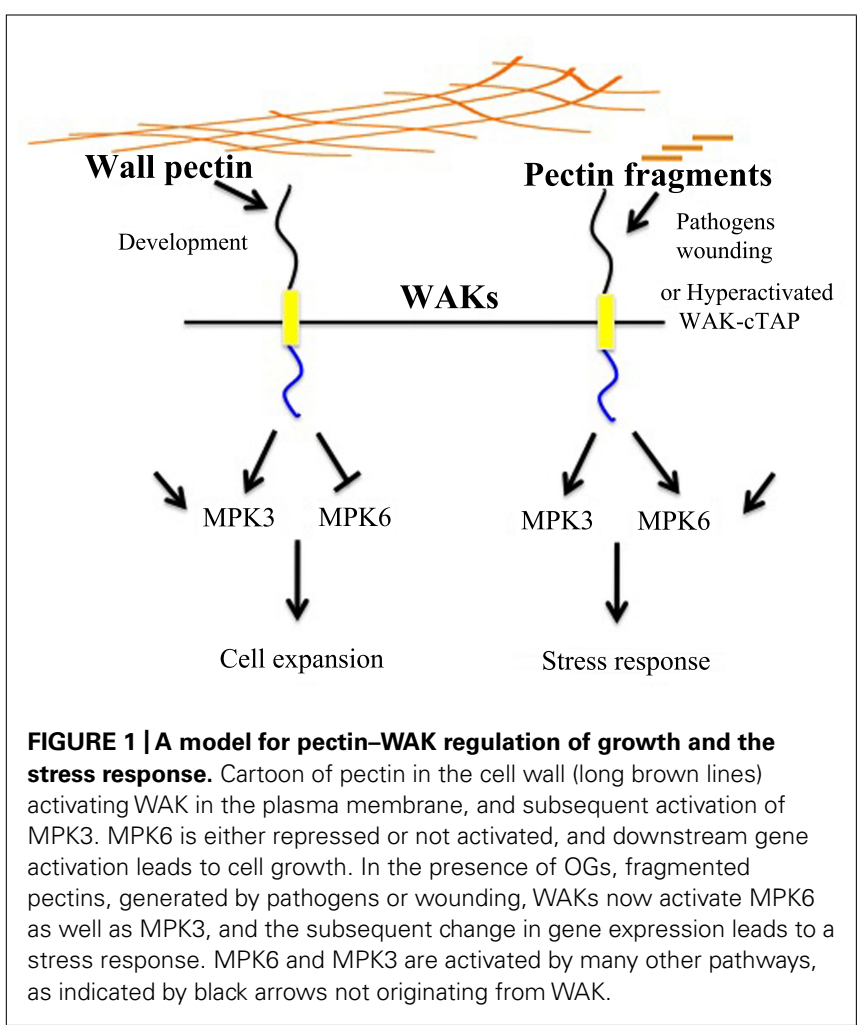




\section{CELL WALL "SENSORS"}

Plant cell walls arise through a complex, developmentally regulated coordination of synthesis, turnover, and interactions between protein and carbohydrates. Screens for mutants in developmental processes have not surprisingly then revealed numerous alleles of cell wall biosynthesis genes, and conversely mutations in cell wall function have identified alleles in genes normally associated with a variety of metabolic and developmental pathways (Seifert and Blaukopf, 2010). These genes include receptor kinases such as THESEUS (THE1; Hematy et al., 2007; Hematy and Hofte, 2008), FERONIA (FER), HERKULES (HERK), and ANXUR (Hematy and Hofte, 2008; Guo et al., 2009a,b; Miyazaki et al., 2009). This class of receptor has been reviewed extensively elsewhere (Seifert and Blaukopf, 2010; Cheung and Wu, 2011) and they have been termed cell wall sensors. However, it may be more accurate at this point to describe them as regulators of pathways that are linked physiologically, biochemically, or physically to aspects of cell wall biology.

The WAKs are like many other plant receptors in that they regulate some aspect of cell division or growth, but also affect

\section{REFERENCES}

Anderson, C. M., Wagner, T. A., Perret, M., He, Z. H., He, D., and Kohorn, B. D. (2001). WAKs: cell wall-associated kinases linking the cytoplasm to the extracellular matrix. Plant Mol. Biol. 47, 197-206.

Andreasson, E., and Ellis, B. (2010). Convergence and specificity in the Arabidopsis MAPK nexus. Trends Plant Sci. 15, 106-113.

Bosch, M., and Hepler, P. K. (2005). Pectin methylesterases and pectin dynamics in pollen tubes. Plant Cell 17, 3219-3226.

Brutus, A., Sicilia, F., Macone, A., Cervone, F., and De Lorenzo, G. (2010). A domain swap approach reveals a role of the plant wall-associated kinase 1 (WAK1) as a receptor of oligogalacturonides. Proc. Natl. Acad. Sci. U.S.A. 107, 9452-9457.

Caffall, K. H., and Mohnen, D. (2009). The structure, function, and biosynthesis of plant cell wall pectic polysaccharides. Carbohydr. Res. 344, 1879-1900.

Cheung, A. Y., and Wu, H. M. (2011). THESEUS 1, FERONIA and relatives: a family of cell wall-sensing receptor kinases? Curr. Opin. Plant Biol. 14, 632-641.

Decreux, A., and Messiaen, J. (2005). Wall-associated kinase WAK1 interacts with cell wall pectins in a calcium-induced conformation. Plant Cell Physiol. 46, 268-278.

Decreux, A., Thomas, A., Spies, B., Brasseur, R., Van Cutsem, P., and Messiaen, J. (2006). In vitro characterization of the homogalacturonan-binding domain of the wall-associated kinase WAK1 using site-directed mutagenesis. Phytochemistry 67, 1068-1079.

Denoux, C., Galletti, R., Mammarella, N., Gopalan, S., Werck, D., De Lorenzo, G., Ferrari, S., Ausubel, F. M., and Dewdney, J. (2008). Activation of defense response pathways by OGs and Flg22 elicitors in Arabidopsis seedlings. Mol. Plant 1, 423-445.

Diener, A. C., and Ausubel, F. M. (2005). RESISTANCE TO FUSARIUM OXYSPORUM 1, a dominant Arabidopsis disease-resistance gene, is not race specific. Genetics 171, 305-321.

Guo, H., Li, L., Ye, H., Yu, X., Algreen, A., and Yin, Y. (2009a). Three related receptor-like kinases are required for optimal cell elongation in Arabidopsis thaliana. Proc. Natl. Acad. Sci. U.S.A. 106, 7648-7653.

Guo, H., Ye, H., Li, L., and Yin, Y. (2009b). A family of receptor-like kinases are regulated by BES1 and involved in plant growth in Arabidopsis thaliana. Plant Signal. Behav. 4, 784-786.

Harholt, J., Suttangkakul, A., and Vibe Scheller, H. (2010). Biosynthesis of pectin. Plant Physiol. 153, 384-395.

He, Z. H., Cheeseman, I., He, D., and Kohorn, B. D. (1999). A cluster of five cell wall-associated receptor kinase genes, Wak1-5, are expressed in specific organs of Arabidopsis. Plant Mol. Biol. 39, 1189-1196.

He, Z. H., Fujiki, M., and Kohorn, B. D. (1996). A cell wall-associated, receptor-like protein kinase. J. Biol. Chem. 271, 19789-19793.

He, Z. H., He, D., and Kohorn, B. D. (1998). Requirement for the induced expression of a cell wall associated

aspects of the cell wall; perhaps WAKs are also cell wall sensors. But WAKs remain unique in that they are known to bind directly to a major structural component of the cell wall, pectin, which appears to influence a WAK-dependent signaling pathway regulating cell expansion. WAKs are also involved in the pathogen and stress responses, but are likely activated in this case by fragmented pectins. An understanding of how WAKs are regulated by the various forms of pectin in cell walls will be greatly aided by a detailed knowledge of their structural requirements for binding pectin, and by an analysis of the distribution of WAKs and pectins in the cell wall. With the realization that the cell wall is so intimately involved in plant development and the pathogen response, and in turn knowing that there are numerous signaling pathways that regulate these events, it would not be surprising to discover that WAKs form networks with numerous other receptor pathways.

\section{ACKNOWLEDGMENTS}

The work was supported by generous funding from the NSF, more recently by MCB 0717983 to Bruce D. Kohorn.

receptor kinase for survival during the pathogen response. Plant J. 14, 55-63.

Hematy, K., and Hofte, H. (2008). Novel receptor kinases involved in growth regulation. Curr. Opin. Plant Biol. 11, 321-328.

Hematy, K., Sado, P. E., Van Tuinen, A., Rochange, S., Desnos, T., Balzergue, S., Pelletier, S., Renou, J. P., and Hofte, H. (2007). A receptorlike kinase mediates the response of Arabidopsis cells to the inhibition of cellulose synthesis. Curr. Biol. 17, 922-931.

Kohorn, B. D. (2000). Plasma membrane-cell wall contacts. Plant Physiol. 124, 31-38.

Kohorn, B. D. (2001). WAKs; cell wall associated kinases. Curr. Opin. Cell Biol. 13, 529-533.

Kohorn, B. D., Johansen, S., Shishido, A., Todorova, T., Martinez, R., Defeo, E., and Obregon, P. (2009). Pectin activation of MAP kinase and gene expression is WAK2 dependent. Plant J. 60, 974-982.

Kohorn, B. D., Kobayashi, M., Johansen, S., Friedman, H. P., Fischer, A., and Byers, N. (2006a). Wall-associated kinase 1 (WAK1) is crosslinked in endomembranes, and transport to the cell surface requires correct cellwall synthesis. J. Cell Sci. 119, 22822290.

Kohorn, B. D., Kobayashi, M., Johansen, S., Riese, J., Huang, L. F., Koch, K., Fu, S., Dotson, A., and Byers, N. (2006b). An Arabidopsis cell wall-associated kinase required for invertase activity and cell growth. Plant J. 46, 307-316. Kohorn, B. D., Kohorn, S. L., Todorova, T., Baptiste, G., Stansky, K., and
McCullough, M. (2011). A dominant allele of Arabidopsis pectin-binding wall-associated kinase induces a stress response suppressed by MPK6 but not MPK3 mutations. Mol. Plant doi: 10.1093/mp/ssr096 [Epub ahead of print].

Kohorn, B. D., Lane, S., and Smith, T. A. (1992). An Arabidopsis serine/threonine kinase homologue with an epidermal growth factor repeat selected in yeast for its specificity for a thylakoid membrane protein. Proc. Natl. Acad. Sci. U.S.A. 89, 10989-10992.

Krichevsky, A., Kozlovsky, S. V., Tian, G. W., Chen, M. H., Zaltsman, A., and Citovsky, V. (2007). How pollen tubes grow. Dev. Biol. 303, 405-420.

Lally, D., Ingmire, P., Tong, H. Y., and He, Z. H. (2001). Antisense expression of a cell wall-associated protein kinase, WAK4, inhibits cell elongation and alters morphology. Plant Cell 13, 1317-1331.

Li, H., Zhou, S. Y., Zhao, W. S., Su, S. C., and Peng, Y. L. (2009). A novel wall-associated receptor-like protein kinase gene, OsWAK1, plays important roles in rice blast disease resistance. Plant Mol. Biol. 69, 337-346.

Mangeon, A., Junqueira, R. M., and Sachetto-Martins, G. (2010). Functional diversity of the plant glycinerich proteins superfamily. Plant Signal. Behav. 5, 99-104.

Miyazaki, S., Murata, T., SakuraiOzato, N., Kubo, M., Demura, T., Fukuda, H., and Hasebe, M. (2009). ANXUR1 and 2, sister genes to FERONIA/SIRENE, are male factors for coordinated fertilization. Curr. Biol. 19, 1327-1331. 
Mohnen, D. (2008). Pectin structure and biosynthesis. Curr. Opin. Plant Biol. 11, 266-277.

Moscatiello, R., Mariani, P., Sanders, D. and Maathuis, F. J. (2006). Transcriptional analysis of calcium-dependent and calcium-independent signalling pathways induced by oligogalacturonides. J. Exp. Bot. 57, 2847-2865.

Mousavi, A., and Hotta, Y. (2005). Glycine-rich proteins: a class of novel proteins. Appl. Biochem. Biotechnol. 120, 169-174.

Park, A. R., Cho, S. K., Yun, U. J., Jin, M. Y., Lee, S. H., Sachetto-Martins, G., and Park, O. K. (2001). Interaction of the Arabidopsis receptor protein kinase Wak1 with a glycine-rich protein, AtGRP-3. J. Biol. Chem. 276, 26688-26693.

Ringli, C., Keller, B., and Ryser, U. (2001). Glycine-rich proteins as structural components of plant cell walls. Cell. Mol. Life Sci. 58, 1430 1441.

Sampoli Benitez, B. A., and Komives, E. A. (2000). Disulfide bond plasticity in epidermal growth factor. Proteins 40, 168-174.

Seifert, G. J., and Blaukopf, C. (2010). Irritable walls: the plant extracellular matrix and signaling. Plant Physiol. 153, 467-478.

Sivaguru, M., Ezaki, B., He, Z. H., Tong, H., Osawa, H., Baluska, F., Volkmann, D., and Matsumoto, H. (2003). Aluminum-induced gene expression and protein localization of a cell wall-associated receptor kinase in Arabidopsis. Plant Physiol. 132 2256-2266.

Verica, J. A., Chae, L., Tong, H., Ingmire, P., and He, Z. H. (2003). Tissue-specific and developmentally regulated expression of a cluster of tandemly arrayed cell wall-associated kinase-like kinase genes in Arabidopsis. Plant Physiol. 133, 1732-1746.

Verica, J. A., and He, Z. H. (2002). The cell wall-associated kinase (WAK) and WAK-like kinase gene family. Plant Physiol. 129, 455-459.

Wagner, T. A., and Kohorn, B. D. (2001). Wall-associated kinases are expressed throughout plant development and are required for cell expansion. Plant Cell 13, 303-318.

Willats, W. G., McCartney, L., Mackie, W., and Knox, J. P. (2001). Pectin: cell biology and prospects for functional analysis. Plant Mol. Biol. 47, 9-27.

Wolf, S., Mouille, G., and Pelloux, J. (2009). Homogalacturonan methylesterification and plant development. Mol. Plant 2, 851-860.

Yamazaki, N., Fry, S. C., Darvill A. G., and Albersheim, P. (1983). Host-pathogen interactions: XXIV. Fragments isolated from suspensioncultured sycamore cell walls inhibit the ability of the cells to incorporate $[\mathrm{C}]$ leucine into proteins. Plant Physiol. 72, 864-869.

Zhang, S., Chen, C., Li, L., Meng, L., Singh, J., Jiang, N., Deng, X. W., He, Z. H., and Lemaux, P. G. (2005). Evolutionary expansion, gene structure, and expression of the rice wallassociated kinase gene family. Plant Physiol. 139, 1107-1124.
Conflict of Interest Statement: The authors declare that the research was conducted in the absence of any commercial or financial relationships that could be construed as a potential conflict of interest.

Received: 13 March 2012; paper pending published: 13 April 2012; accepted: 18 April 2012; published online: 08 May 2012.

Citation: Kohorn BD and Kohorn SL (2012) The cell wall-associated kinases, WAKs, as pectin receptors. Front. Plant Sci. 3:88. doi: 10.3389/fpls.2012.00088

This article was submitted to Frontiers in Plant Physiology, a specialty of Frontiers in Plant Science.

Copyright (c) 2012 Kohorn and Kohorn.

This is an open-access article distributed under the terms of the Creative Commons Attribution Non Commercial License, which permits non-commercial use, distribution, and reproduction in other forums, provided the original authors and source are credited. 